\title{
Práticas de grupo desenvolvidas pelo núcleo ampliado de saúde da família e atenção básica: uma revisão integrativa da literatura
}

Group practices developed by the expanded nucleus of family health and basic care: an integrative

\author{
literature review
}

Prácticas de grupo desarrolladas por el núcleo ampliado de salud familiar y atención básica: una

revisión integrativa de la literatura

Recebido: 08/12/2021 | Revisado: 12/12/2021 | Aceito: 13/12/2021 | Publicado: 21/12/2021

Jainne Oliveira Leão Silva ORCID: https://orcid.org/0000-0002-4796-083X

Faculdade Independente do Nordeste, Brasil E-mail: jainneleao2015@gmail.com

Jaianne Oliveira Leão Silva

ORCID: https://orcid.org/0000-0002-8741-3128

Faculdade Independente do Nordeste, Brasil

E-mail: jaianneleao@gmail.com

Rangel Campos Pereira

ORCID: https://orcid.org/0000-0001-5776-4771

Centro Universitário Faculdade de Tecnologia e Ciência, Brasil E-mail: rangelcampos36@gmail.com

Marcos Vinicius Rodrigues Oliveira Silva

ORCID: https://orcid.org/0000-0001-5011-3378

Centro Universitário Faculdade de Tecnologia e Ciência, Brasil

E-mail: viniciusrohdrigues@gmail.com

Erik das Merces Santos

ORCID: https://orcid.org/0000-0002-8741-3128

Centro Universitário Faculdade de Tecnologia e Ciência, Brasil

E-mail: erikcerti@gmail.com

Gabriele Lima Nunes

ORCID: https://orcid.org/0000-0002-3072-921X

Centro Universitário Faculdade de Tecnologia e Ciência, Brasil

E-mail: gabrielelimanunes31@gmail.com

Grazyelle Teles Brito

ORCID: https://orcid.org/0000-0002-9304-6416

Centro Universitário Faculdade de Tecnologia e Ciência, Brasil

E-mail: grazyelle.brito@ftc.edu.br

André de Oliveira Lacerda

ORCID: https://orcid.org/0000-0002-6112-116X

Centro Universitário Faculdade de Tecnologia e Ciência, Brasil

E-mail: andre.lacerda@live.com

Lázaro Silva Sousa

ORCID: https://orcid.org/0000-0003-2297-2831

Centro Universitário Faculdade de Tecnologia e Ciência, Brasil

E-mail: lazarosousa05@gmail.com

Jean Sousa Almeida

ORCID: https://orcid.org/0000-0003-3911-9650

Centro Universitário Faculdade de Tecnologia e Ciência, Brasil

E-mail: jean.so.almeida@gmail.com

Alan Agnelo Bonfim da Silva

ORCID: https://orcid.org/0000-0001-5161-9960

Centro Universitário Faculdade de Tecnologia e Ciência, Brasil

E-mail: alanagnello89@gmail.com

Luanna Evelyn Inácio Pessoa

ORCID: https://orcid.org/0000-0002-2509-2656

Centro Universitário Faculdade de Tecnologia e Ciência, Brasil

E-mail: luapessoafisio@gmail.com

Talita Santos Oliveira Sampaio

ORCID: https://orcid.org/0000-0002-6351-1321

Faculdade Independente do Nordeste, Brasil E-mail: talitafisio@gmail.com 


\section{Resumo}

Este estudo objetiva descrever o processo de trabalho do núcleo ampliado de saúde da família e atenção básica com ênfase nas práticas de grupos desenvolvidas pelos profissionais do núcleo ampliado de saúde da família e atenção básica. Trata-se de uma revisão integrativa com base na pesquisa avançada de estudos nas bases de dados: SCIELO, PubMed e LILACS, utilizando os operadores boleanos AND e OR. Foram incluídos artigos publicados em língua portuguesa que retratassem a respectiva temática, entre os períodos de 2011 a 2021 e excluídos aqueles com mais de dez anos, que não estivessem disponíveis gratuitamente e os que não estavam associados ao presente tema, monografias, dissertação, teses, resumos e artigos duplicados. Foram selecionados 09 artigos pelos critérios de inclusão previamente estabelecidos. Identificou-se grupos com grande variedade de públicos alvo. Conclui-se que, as práticas de grupo realizadas pelas equipes do Núcleo Ampliado de Saúde da Família podem beneficiar inúmeras pessoas, tendo a possibilidade de desenvolver grupos em diversas áreas e temas de acordo com as necessidades específicas de cada população. Entretanto, houve dificuldade na busca dos artigos, pelo fato de que inúmeros autores apenas citaram o desenvolvimento dos grupos, sem detalhar sua finalidade e quais atividades eram realizadas em cada grupo, deixando uma lacuna que dificulta e compromete o entendimento acerca do processo de trabalho do núcleo ampliado de saúde da família e atenção básica, no âmbito dos grupos terapêuticos/educativos.
\end{abstract}

Palavras-chave: Educação em saúde; Prática de grupo; Saúde coletiva.

\begin{abstract}
This study aims to describe the work process of the extended family health and primary care nucleus, with an emphasis on group practices developed by professionals from the extended family health and primary care nucleus. This is an integrative review based on advanced research of studies in databases: SCIELO, PubMed and LILACS, using the Boolean operators AND and OR, using the keywords: NASF, collective activity, continuing education, education in health and group practice. Articles published in Portuguese that portrayed the respective theme were included, between the periods 2011 to 2021, and those older than ten years old, that were not available for free and those that were not associated with this theme, monographs, dissertation, theses, were excluded. abstracts and duplicate articles. 09 articles were selected according to the previously established inclusion criteria. Groups with a wide variety of target audiences were identified. It is concluded that the group practices carried out by the teams of the Expanded Family Health Center can benefit countless people, with the possibility of developing groups in different areas and themes according to the specific needs of each population. However, the search for articles was difficult, due to the fact that numerous authors only cited the development of the groups, without detailing their purpose and what activities were carried out in each group, leaving a gap that hinders and compromises the understanding of the work process of the expanded nucleus of family health and primary care, within the scope of therapeutic/educational groups.
\end{abstract}

Keywords: Health education; Group practice; Collective health.

\title{
Resumen
}

Este estudio tiene como objetivo describir el proceso de trabajo del núcleo de salud familiar extendida y atención primaria, con énfasis en las prácticas grupales desarrolladas por profesionales del núcleo de salud familiar extendida y atención primaria. Se trata de una revisión integradora basada en la investigación avanzada de estudios en bases de datos: SCIELO, PubMed y LILACS, utilizando los operadores booleanos AND y OR, utilizando las palabras clave: NASF, actividad colectiva, educación continua, educación en salud y práctica grupal. Se incluyeron artículos publicados en portugués que retrataban la temática respectiva, entre los períodos 2011 a 2021, y aquellos mayores de diez años, que no estaban disponibles de forma gratuita y aquellos que no estaban asociados a esta temática, monografías, disertaciones, tesis, fueron excluidos resúmenes y artículos duplicados. Se seleccionaron 09 artículos según los criterios de inclusión previamente establecidos. Se identificaron grupos con una amplia variedad de públicos objetivo. Se concluye que las prácticas grupales que realizan los equipos del Centro Ampliado de Salud de la Familia pueden beneficiar a innumerables personas, con la posibilidad de desarrollar grupos en diferentes áreas y temáticas de acuerdo a las necesidades específicas de cada población. Sin embargo, la búsqueda de artículos fue difícil, debido a que numerosos autores solo citaron el desarrollo de los grupos, sin detallar su propósito y qué actividades se realizaban en cada grupo, dejando una brecha que dificulta y compromete la comprensión del trabajo. proceso del núcleo ampliado de salud de la familia y atención primaria, en el ámbito de los grupos terapéuticos / educativos.

Palabras clave: Educación para la salud; Práctica grupal; Salud pública.

\section{Introdução}

No Brasil, o sistema que orienta a saúde de sua população é o Sistema Único de Saúde (SUS), que tem como objetivo, garantir a saúde como direito do cidadão e dever do Estado (Brasil, 1990). Atrelado a esse sistema, foi desenvolvido a Estratégia de Saúde da Família (ESF) a fim de ser o primeiro contato do usuário nesta rede assistencial, atuando na atenção básica de forma integrada (Brasil, 2011; Quirino, 2020). 
Objetivando ampliar ações da Atenção Básica e sua resolutividade, bem como, prestar assistência à alta demanda populacional assistida pelas equipes de saúde da família, o Ministério da Saúde, mediante a portaria n ${ }^{\circ} 154$, em 2008, criou o Núcleo de Apoio à Saúde da Família (NASF). Posteriormente, em 2017, por meio da Portaria nº 2.436, houve a revisão de diretrizes para a organização da Atenção Básica e com isso, o NASF passou por algumas modificações, dentre elas, sua nomenclatura, passando a ser o Núcleo Ampliado de Saúde da Família e Atenção Básica (Brasil, 2008; Brasil, 2012; Diário Oficial da União, 2017).

Dentre os profissionais que atuam no Núcleo Ampliado de Saúde da Família e Atenção Básica (NASF-AB), pode-se encontrar o fisioterapeuta, psicólogo, assistente social, farmacêutico, fonoaudiólogo, profissional de educação física e etc (Diário Oficial da União, 2017; Brasil, 2012). Entretanto, os profissionais eleitos para compor as equipes, são definidos pelos gestores municipais em função das necessidades de saúde, vulnerabilidades socioeconômicas e perfil epidemiológico da comunidade. Sendo, a proposta de trabalho das equipes do NASF-AB, transcender aos modelos convencionais de assistência de trabalho fragmentado e individual, onde priorizava-se a quantidade e não a qualidade, baseando-se na construção de uma rede de atenção integral, longitudinal e resolutiva que articule os diferentes níveis assistenciais (do Nascimento \& de Campos Oliveira, 2010).

O processo de trabalho das equipes do NASF-AB envolve a atuação multidisciplinar, integralidade do cuidado, humanização, educação em saúde, alimentação e nutrição, práticas integrativas e complementares, saúde mental, reabilitação, serviço social, assistência farmacêutica, construção de Projetos Terapêuticos Singulares (PTS), apoio matricial, entre outros (Quirino et al., 2020; Freire et al., 2020).

Estudos analisam que a qualidade de vida de indivíduos que participam das atividades oferecidas pelo NASF$\mathrm{AB}$ e dos que não participam, é significativamente diferente no que se refere a limitação por aspectos físicos, sugerindo um impacto positivo na saúde dos usuários que participam dessas atividades. É notável que a realização das práticas em grupo são consideradas uma importante estratégia de assistência à saúde, contribuindo para melhorias nos hábitos de vida e saúde dos indivíduos, bem estar e favorecem o vínculo entre usuário e profissional do NASF-AB (Tortoro et al 2017; Fernandes, Souza \& Rodrigues; 2019).

Observa-se, porém, que apesar de todos os benefícios e amplo processo de trabalho das equipes de NASF-AB por todo o país, há uma limitação da percepção da população a respeito das atividades prestadas, impossibilitando muitas vezes a assistência e a busca do usuário ao serviço. Há fatores e condições que tendem a dificultar este processo, tais como a pouca oferta das equipes em algumas regiões, e naquelas que existem observam-se poucos profissionais atuando. Além disso, a falta de comunicação entre as equipes do NASF-AB e ESF na busca de discussões sobre as atividades e a pouca capacitação dos profissionais também se tornam um empecilho para uma atuação completa e efetiva do NASF-AB (Souza; Medina, 2018). Nota-se em contrapartida que, os usuários que têm conhecimento sobre esse trabalho, revelam uma forma de ressignificação do conceito de qualidade de vida, autocuidado e promoção de saúde (Souza et al., 2013; Aciole \& Oliveira, 2017; Souza \& Medina, 2018).

Diante dessas considerações esta pesquisa apresenta como objetivo, descrever o processo de trabalho do núcleo ampliado de saúde da família e atenção básica com ênfase nas práticas de grupos desenvolvidas pelos profissionais do núcleo ampliado de saúde da família.

\section{Metodologia}

Trata-se de uma revisão integrativa, que, segundo De Souza et al. (2017), é um método capaz de reunir e sintetizar o conhecimento e a experiência de diversos autores e incorporar em um único trabalho, favorecendo a compreensão mais abrangente de um determinado fenômeno, bem como a obtenção de novas análises através da conjugação dos respectivos 
resultados. Inclusive, Marconi e Lakatos (2003), complementam que a pesquisa bibliográfica não se resume a mera repetição do que já foi dito ou escrito sobre certo assunto, mas proporciona um novo enfoque ou abordagem, chegando a conclusões inovadoras.

Os estudos utilizados para fundamentar essa pesquisa foram selecionados a partir da busca avançada nas bases de dados: Scientific Electronic Library Online (SCIELO), PubMed e Literatura Latino-Americana e do Caribe em Ciências da Saúde (LILACS), utilizando os operadores boleanos AND e OR, da seguinte forma: NASF AND atividade coletiva, NASF AND prática de grupo, NASF AND educação permanente, NASF AND Educação em saúde, NASF AND collective activity, NASF AND group practice, NASF Permanent Education e NASF AND Health education.

Os critérios de inclusão definidos para a seleção dos artigos foram: artigos publicados em língua portuguesa que retratassem a respectiva temática, indexados nos referidos bancos de dados entre os períodos de 2011 a 2021. A seleção se deu por meio da leitura dos títulos e resumos e, após, a leitura na íntegra para observar os critérios de inclusão e exclusão.

Os artigos excluídos foram todos aqueles com mais de dez anos contados até o momento que não estivessem disponíveis gratuitamente, bem como aqueles que não estavam diretamente associados ao presente tema, monografias, dissertação, teses, resumos e artigos duplicados.

Figura 1. Delimitação da pesquisa e os resultados encontrados.

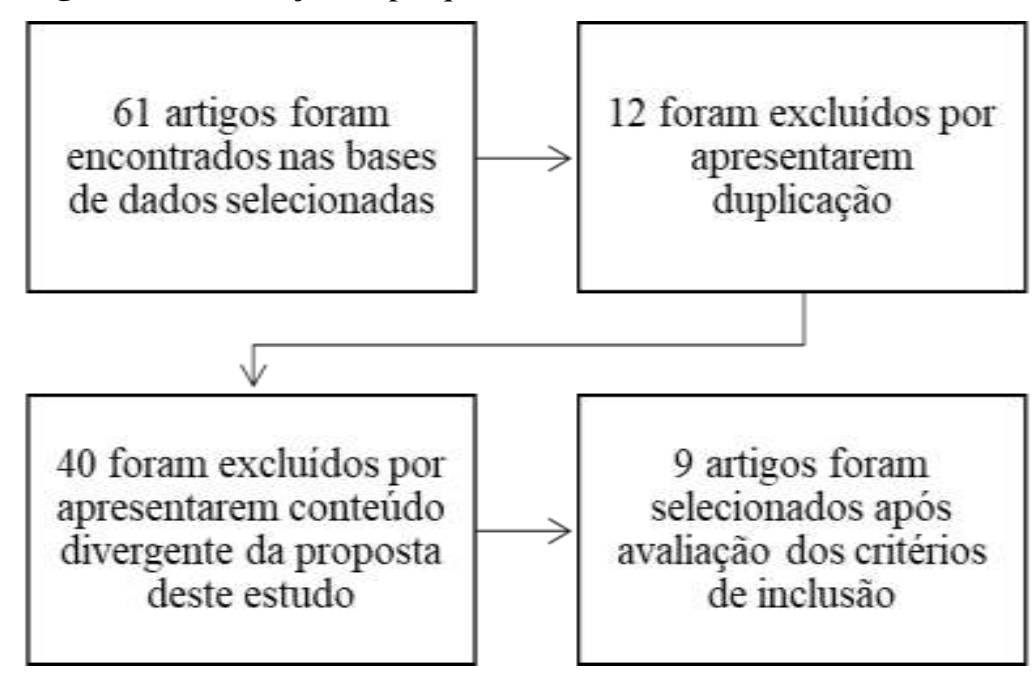

Fonte: Autores (2021).

\section{Resultados}

Na Tabela 1 estão expostos os 9 artigos selecionados mediante a busca avançada nas bases de dados: Scientific Electronic Library Online (SCIELO), PubMed e Literatura Latino-Americana e do Caribe em Ciências da Saúde (LILACS) citadas previamente e seguindo os critérios de inclusão e exclusão previamente estabelecidos e organizados na tabela por autor e ano de publicação, título, tipo do estudo/amostra e resultados principais. 
Tabela I- Elementos de identificação dos trabalhos selecionados após aplicação dos critérios de inclusão e exclusão.

\begin{tabular}{|c|c|c|}
\hline $\begin{array}{c}\text { AUTORES E } \\
\text { ANO }\end{array}$ & $\begin{array}{c}\text { TÍTULO DO } \\
\text { ARTIGO }\end{array}$ & TIPO DE ESTUDO/AMOSTRA \\
\hline $\begin{array}{c}\text { ARCE; } \\
\text { TEIXEIRA } \\
(2017)\end{array}$ & $\begin{array}{l}\text { Práticas de saúde e } \\
\text { modelo de atenção } \\
\text { no âmbito do } \\
\text { Núcleo de Apoio à } \\
\text { Saúde da Família } \\
\text { em Salvador (BA). }\end{array}$ & $\begin{array}{l}\text { Pesquisa qualitativa do tipo estudo de caso } \\
\text { sobre o processo de trabalho dos } \\
\text { profissionais dos Nasf de Salvador (BA). } \\
\text { Compõem estas equipes profissionais de } \\
\text { educação física, fisioterapia, nutrição, } \\
\text { psicologia, serviço social e terapia } \\
\text { ocupacional. }\end{array}$ \\
\hline $\begin{array}{c}\text { DOS SANTOS, } \\
\text { DOMINGOS, } \\
\text { BRAGA, SPIRI } \\
(2018)\end{array}$ & $\begin{array}{lr}\text { Saúde mental na } \\
\text { atenção } & \text { básica: } \\
\text { experiência } & \text { de } \\
\text { matriciamento } & \text { na } \\
\text { área rural } & \end{array}$ & $\begin{array}{l}\text { Estudo do tipo relato da experiência, } \\
\text { desenvolvido em um município localizado } \\
\text { no centro-oeste do estado de São Paulo, } \\
\text { sob cobertura de } 11 \text { unidades da ESF. } \\
\text { Baseado nos pontos frágeis do processo de } \\
\text { trabalho, as equipes da USF e do NASF } \\
\text { realizaram um planejamento para } \\
\text { redirecionar e reorganizar as ações de cada } \\
\text { trabalhador da equipe da unidade }\end{array}$ \\
\hline
\end{tabular}

$\begin{array}{cll}\text { NOGUEIRA, } & \text { Planejamento local } \\ \text { LABEGALINI, } & \text { de saúde: atenção ao } \\ \text { CARREIRA, } & \text { idoso versus } \\ \text { BALDISSERA } & \text { Educação vere em } \\ \text { (2018) } & \begin{array}{l}\text { Permanente } \\ \text { Saúde }\end{array}\end{array}$

Pesquisa com abordagem qualitativa, exploratória-descritiva, analíticointerpretativo, realizada no período de agosto a outubro de 2017, que utilizou como fonte de dados os planos locais de saúde das UBSs de um município localizado na região Noroeste do estado do Paraná, Brasil. Obteve-se 29 planos focais

$\begin{array}{cl}\text { NOGUEIRA; } & \text { Atenção ao idoso: } \\ \text { ACIOLI; } & \text { práticas de educação } \\ \text { CARREIRA; } & \text { permanente do } \\ \text { BALDISSERA } & \text { Núcleo de Apoio à } \\ \text { (2019) } & \text { Saúde da Família }\end{array}$

FERNANDES; Práticas de grupo do

SOUZA; Núcleo de Apoio à

RODRIGUES

(2019)

Saúde da Família: perspectiva do usuário
Pesquisa qualitativa, de campo, do tipo exploratório-descritiva de caráter analítico, interpretativo. Desenvolvido em um município do estado do Paraná com 46 profissionais integrantes nas equipes de nove NASF do estado, de fevereiro a abril de 2017, por meio da técnica de Grupo Focal

Estudo exploratório, qualitativo, realizado no município de Governador Valadares (MG) com 27 usuários do NASF.

\section{(Atividades em grupo desenvolvidas pelo NASF)}

As "atividades clínicas voltadas para os usuários" foram as mais citadas, observando-se o predomínio de atendimentos clínicos de curta duração. Também é significativa a realização de "atividades educativas de prevenção".

Identificou-se a escassez de ações dos grupos e cuidado coletivo. Durante as reuniões, ocorreram ações de sensibilização sobre o uso de psicofármacos e o cuidado em saúde junto aos trabalhadores.

Quanto às ações de cuidado coletivo, havia o grupo para cessar o tabagismo e como estratégia de intervenção, foram planejadas atividades de Educação em Saúde na lógica de grupos. Nos encontros, foram abordados temas de interesse dos participantes: definição e função dos psicofármacos; diferença entre ansiolíticos e antidepressivos; cuidados na armazenagem dos medicamentos; importância no uso correto; práticas integrativas e complementares; qualidade do sono; atividade física e lazer; medicações fitoterápicas; uso de chás e dependência farmacológica, por meio de rodas de conversa, práticas de relaxamento e alongamento, oficina de preparo de chás, dança, atividades aeróbicas, vídeos ilustrativos e dinâmicas interpessoais.

As ações programadas de EPS na atenção ao idoso apresentaram enfoque às ações desempenhadas pelos profissionais do NASF enquanto promotor de atividades educativas, assistenciais e na gestão de casos, seguido dos grupos operativos, sendo esta, voltada para a prevenção de doenças e agravos, manutenção e promoção da saúde.

Observou-se que a EPS na atenção ao idoso é reduzida à temática de prevenção de quedas na terceira idade, onde são desenvolvidas ações por meio de orientações aos idosos e seus cuidadores.

A EPS se dá pela discussão de casos que ocorrem principalmente durante reuniões das equipes e nas consultas compartilhadas, pelo matriciamento, na realização da Visita Domiciliar, nas práticas desenvolvidas durante os grupos educativos voltados à promoção de saúde e prevenção de agravos, com destaque para os grupos de atividade física e de pessoas com condições crônicas (HIPERDIA) grupo de cuidadores de idosos, artesanato.

Neste município, o NASF desenvolve diversas práticas de grupo voltadas para a prevenção e promoção da saúde, contemplando ações como: hidroginástica, caminhadas orientadas, alongamentos, ginástica localizada, ginástica aeróbica, zumba, treinamento funcional, grupos de hipertensos e diabéticos, grupos de homens, grupos de saúde mental, grupos de emagrecimento e orientação nutricional, grupos de artesanato/geração de renda.

\begin{tabular}{clr}
\hline VENDRUSCOL & Características e \\
O, METELSKI, & atuação dos do d \\
MAFFISSONI, & profissionais dos \\
TESSER, & Núcleos Ampliados \\
TRINDADE & de Saúde da Família \\
(2019) & e Atenção Básica
\end{tabular}

Pesquisa descritiva cuja produção e análise dos dados foi utilizada a abordagem quanti-qualitativa, estratégia exploratória e sequencial, ocorreu no estado de Santa Catarina. Os dados foram obtidos em duas etapas, na etapa QUAN, 359 nasfianos responderam a um instrumento Survey, com 19 questões e na etapa QUAL, participaram 43 nasfianos, onde passaram por entrevistas entre setembro a novembro de 2017.

\begin{tabular}{|c|c|c|}
\hline $\begin{array}{l}\text { NOVAES, } \\
\text { WANDERLEY, } \\
\text { FALCÃO, } \\
\text { ALVES, LIMA, } \\
\text { SOARES (2020) }\end{array}$ & $\begin{array}{l}\text { Protocolo de } \\
\text { atividade física } \\
\text { remoto para grupos } \\
\text { de Academia da } \\
\text { Saúde e Estratégia } \\
\text { de Saúde da Família }\end{array}$ & $\begin{array}{l}\text { O estudo consiste em uma investigação- } \\
\text { ação, desenvolvida no âmbito das } \\
\text { Academias da Saúde e equipes de } \\
\text { NASF-AB do município de Arapiraca, } \\
\text { Alagoas. }\end{array}$ \\
\hline
\end{tabular}

As atividades coletivas/grupos produzidos nos Nasf-AB, sejam específicos ou não, a partir da área de formação do nasfiano e são realizadas diariamente e até três vezes por semana, sendo os grupos de reeducação alimentar e grupos de Desenvolvimento Humano.

O programa de Prática Corporal/Atividade Física remota foi denominado "Agite em Casa". Deu início em maio de 2020 como estratégia de reativação dos grupos de atividade física das Academias da Saúde e dos NASF-AB, considerando o plano de contingência para SARS-CoV-2. A base de exercícios criada, contem 53 vídeos no total, com exercícios para membros superiores, inferiores, alongamentos dinâmicos e estáticos, aquecimento, exercícios aeróbicos e exercícios voltados para a musculatura do tronco. 


\begin{tabular}{|c|c|c|c|}
\hline $\begin{array}{c}\text { SILVA; } \\
\text { NOGUEIRA; } \\
\text { SILVA; } \\
\text { FROTA }(2020)\end{array}$ & $\begin{array}{l}\text { Atuação do } \\
\text { fisioterapeuta nos } \\
\text { núcleos de apoio à } \\
\text { saúde da família em } \\
\text { Teresina, Piauí }\end{array}$ & $\begin{array}{l}\text { Estudo descritivo, transversal e } \\
\text { quantitativo, que teve como sujeitos todos } \\
\text { os fisioterapeutas (6) vinculados às } \\
\text { equipes do Núcleo de Apoio à Saúde da } \\
\text { Família, localizados na cidade de Teresina } \\
(\text { PI). }\end{array}$ & $\begin{array}{l}\text { Dentre as atividades desenvolvidas pelos profissionais } \\
\text { entrevistados, há a realização de atendimento em grupo na UBS, } \\
\text { a respeito da saúde da mulher, um dos grupos beneficiados em } \\
\text { relação às orientações posturais é o grupo de gestantes, também } \\
\text { são realizadas atividades relacionadas à saúde do idoso. Além } \\
\text { disso, todos citaram que desenvolvem ações preventivas e } \\
\text { educação em saúde. }\end{array}$ \\
\hline $\begin{array}{l}\text { BIM; } \\
\text { CARVALHO; } \\
\text { TRELHA; } \\
\text { RIBEIRO; } \\
\text { BADUY; } \\
\text { GONZALÉZ } \\
\text { (2021) } \\
\end{array}$ & $\begin{array}{l}\text { Práticas } \\
\text { fisioterapêuticas } \\
\text { para a produção do } \\
\text { cuidado na atenção } \\
\text { primária à saúde }\end{array}$ & $\begin{array}{l}\text { Estudo qualitativo, com referencial } \\
\text { metodológico de análise do discurso, } \\
\text { realizado em Londrina, em agosto de } \\
\text { 2017, com } 19 \text { fisioterapeutas que atuavam } \\
\text { na UBS. }\end{array}$ & $\begin{array}{l}\text { Grupos são outra prática fisioterapêutica na APS, e os mais } \\
\text { frequentes são os grupos de dor e de alongamento. Os demais } \\
\text { grupos variam de uma unidade para outra nas temáticas, sendo } \\
\text { alguns deles interprofissionais: tabagismo, obesidade, ombro, } \\
\text { coluna, gestantes, fortalecimento, isostretching, dança e } \\
\text { auriculoterapia. Alguns grupos são semanais e outros em } \\
\text { módulos. }\end{array}$ \\
\hline
\end{tabular}

Fonte: Dados da pesquisa.

\section{Discussão}

Os grupos educativos e terapêuticos realizados pelas equipes do Núcleo Ampliado de Saúde da Família, são uma importante estratégia para trabalhar a educação em saúde com a população, bem como a promoção da saúde e prevenção de doenças, fortalecendo e favorecendo o vínculo, o acolhimento, o desenvolvimento da autonomia dos usuários, auxiliando na redução da incidência de doenças e de complicações associadas a elas. Essa ferramenta de trabalho abrange diversos públicos alvos, como os grupos de gestantes, idosos, portadores de doenças crônicas como hipertensão arterial sistêmica e diabetes mellitus, grupos de saúde mental e de práticas corporais (Bezerra et al., 2020; Mendonça \& Nunes, 2015).

Em estudo, Arce e Teixeira (2017) observaram que as atividades educativas de prevenção eram conduzidas tanto pela equipe de saúde da família (controle do tabagismo, hiperdia, grupo de idosos e gestantes), quanto pela equipe do NASF-AB (grupo de atividades físicas, controle da obesidade e saúde mental), ou atividades pontuais (salas de espera, feiras de saúde e campanhas sanitárias promovidas pela secretaria municipal de saúde).

Além disso, Sauer e colaboradores (2018) reforçam que além das atividades tradicionais que são realizadas nos grupos, outras atividades podem ser agregadas, como um meio de facilitar a adesão dos usuários aos grupos, a exemplo disso, nos grupos de controle da obesidade, pode-se oferecer oficinas de culinária que facilitam a alimentação adequada, bem como a formação de um grupo de caminhada, como incentivo para a prática da atividade física. Com relação aos grupos de saúde mental, as autoras Silva, Beirão e Dias (2018) relataram a experiência com a utilização da auriculoterapia e terapia floral em um grupo de saúde mental, com encontros semanais de 2 horas. Em ambas terapias foram observados relatos de diminuição ou desaparecimento das dores físicas, tranquilidade, melhora na insônia, tristeza, ansiedade e angustia.

O trabalho acerca do cuidado na saúde mental é complexo e desafiador, entretanto, é notável a sua importância, principalmente devido à alta prevalência de transtornos mentais, neurológicos e por uso abusivo de drogas em todas as regiões do mundo e por serem fatores que contribuem para a morbidade e mortalidade prematuras (Hori \& Nascimento, 2014). As atividades em grupo podem ser realizadas nas escolas, conforme preconiza o Programa Saúde na Escola (PSE), bem como, em grupos voltados para mulheres no intuito de oferecer suporte e apoio, grupos operativos como os que objetivam cessar/reduzir do uso do tabaco, controle da hipertensão e da diabetes, contribuindo na melhoria da qualidade de vida (Alves \& Bruning, 2019).

Dessa forma, Dos Santos et al. (2018) evidenciam a formulação de grupos de saúde mental na atenção básica, onde é identificada a escassez de ações dos grupos e cuidado coletivo. Com relação a este último, havia o grupo para cessar o tabagismo, tendo como intervenção: reuniões para a sensibilização sobre o uso de psicofármacos, possíveis mudanças na aquisição de medicamentos, definição e função dos psicofármacos, diferença entre ansiolíticos e antidepressivos, cuidados na armazenagem dos medicamentos, práticas integrativas e complementares, qualidade do sono, atividade física e lazer, 
medicações fitoterápicas, uso de chás e dependência farmacológica, por meio de rodas de conversa, práticas de relaxamento e alongamento, oficina de preparo de chás, dança circular, atividades aeróbicas, vídeos ilustrativos e dinâmicas interpessoais.

Os grupos de saúde mental realizados no município Jaboatão dos Guararapes, eram realizadas atividades para o reconhecimento de ferramentas com o propósito de lidar com as diversas situações cotidianas, bem como práticas integrativas complementares em saúde, atividades de relaxamento, autocuidado e reflexão (de Moura et al., 2020). Em contrapartida, no estudo de Minozzo e colaboradores (2012) onde destaca-se o Programa de Atendimento às Psicoses, criado com o intuito de evitar os prejuízos das internações hospitalares prolongadas e promover o acompanhamento desses indivíduos pelas equipes de atenção primária de saúde, posteriormente, criou-se o "Grupo dos Nervosos" a fim de oferecer um atendimento integral, onde predominavam indivíduos com transtorno mental, egressas das internações psiquiátricas e de pessoas que necessitavam de medicação psicotrópica.

Além disso, destaca-se a importância em trabalhar de forma efetiva a saúde mental nos grupos de idosos, tal como declara o Plano Internacional de Ação sobre o Envelhecimento (PIAE), que prevê a execução de estratégias que favoreçam a prevenção de transtornos mentais, a descoberta precoce, o tratamento dessas doenças, capacitação de profissionais que atendam esse público, entre outros. Inclusive, a população de idosos são caracterizados como grupo de risco para a depressão e outras alterações de ordem menta, com isso, os grupos temáticos ou terapêuticos, mediante ações de promoção da saúde mental, é capaz de promover a o vínculo entre os participantes e profissionais, socialização de emoções, melhora da autoestima e manejo das próprias emoções (Leandro-França \& Giardini Murta, 2014).

Resultado disso, pode ser observado no estudo desenvolvido por Nogueira et al., (2018), onde salienta-se a importância de ações voltadas a atenção ao idoso por meio de ações de Educação Permanente em Saúde (EPS) desenvolvidas pelo NASF-AB, assim, os autores observaram que as ações programadas na atenção ao idoso se desenvolvem a partir de ações coletivas que ocorrem durante os grupos (prevenção de doenças e agravos, manutenção e promoção da saúde) e individualmente (enfoque domiciliar para idosos acamados, na prevenção de quedas, mediante orientações aos idosos e seus cuidadores, ações centradas nas condições do envelhecimento e acompanhamento desses idosos).

No entanto, uma nova vertente sobre as ações em grupo desenvolvidas com foco na saúde do idoso foram identificadas no estudo conduzido por Gomes e Tonello (2019), realizado com indivíduos de ambos os sexos, entre 35 a 75 anos de idade e que participaram das atividades fisioterapêuticas realizadas nos grupos e propostas pelo NASF nas Unidades de Atenção Primária à Saúde (UAPS) na cidade de Presidente Olegário/MG. As atividades envolveram exercícios de amplitude de movimento, alongamentos e fortalecimentos globais e orientações quanto à postura adotada em diversas atividades diárias. Os usuários foram reavaliados após 10 e 15 sessões em grupo, e foi identificado que a intervenção promoveu aumento da flexibilidade da cadeia muscular posterior e diminuição de dor musculoesquelética em adultos e idosos.

Resultados de Nogueira et al. (2019) explanam que ações de educação permanente com foco na atenção ao idoso realizadas pelo NASF, são desenvolvidas durante os grupos educativos são voltados à promoção de saúde e prevenção de agravos, com destaque para os grupos de atividade física, grupo de pessoas com condições crônicas (HIPERDIA), grupo de cuidadores de idosos, artesanato, entre outros. Torna-se relevante destacar que, os usuários desses grupos não devem ser restritos, ou seja, pessoas saudáveis também podem participar dos grupos de hiperdia, como uma forma de se obter informações valiosas sobre a forma de prevenir estas doenças, da mesma forma que uma pessoa tem o direito de participar em mais de um grupo (Brasil, 2018).

Com ênfase nos grupos de hiperdia, o estudo de Serpa et al. (2018) descreve atividades realizadas com esse público no município de João Pessoa-PB, como: dinâmicas (com objetivo de conhecer o cotidiano dos participantes, suas motivações e prazeres), atividade musical (que buscava responder as questões: o que significa ter uma vida boa pra você? E o que acham que é necessário para tê-la?). Também era realizada a dinâmica que envolvia os hábitos (para discussão e reflexão acerca dos 
hábitos saudáveis ou não, suas percepções e conceitos), bem como a leitura do poema: Recomeçar de Carlos Drummond de Andrade (a fim de favorecer a reflexões sobre como lidar com a frustração e a culpa e a importância da motivação para o autocuidado), outras atividades envolviam a automassagem, alongamentos, técnica de respiração diafragmática ou profunda, roda de conversa sobre descanso e sono e café da manhã diet, para compartilhar cardápios e receitas diet e dialogar sobre alimentação saudável.

Em outra vertente, o estudo de Fernandes et al. (2019), retrata as atividades realizadas pelos grupos conduzidos pelas equipes do NASF, com práticas de grupo voltadas para a prevenção e promoção da saúde, mediante práticas de hidroginástica, caminhadas orientadas, alongamentos, ginástica localizada e aeróbica, zumba, treinamento funcional, grupos de hipertensos e diabéticos, grupos de homens, grupos de saúde mental, grupos de emagrecimento e orientação nutricional, grupos de artesanato/geração de renda.

Achado semelhante foi encontrado no estudo de Bezerra (2021), realizado no grupo de idosos da cidade de Jardim de Seridó - RN, o resultado deste estudo mostrou que as atividades físicas mais prazerosas que o NASF desenvolvia, segundo os usuários dos grupos eram: ginástica e o alongamento, caminhada, dança e atividades recreativas, além disso, 100\% dos participantes relataram que as atividades realizadas nos grupos contribuíram significativamente na qualidade de vida.

O estudo de Vendruscolo e colaboradores (2019) traz a criação de novos grupos, são eles: os grupos de reeducação alimentar, grupos por determinados tipos de patologia e Grupos de Desenvolvimento Humano (GDH). Em conformidade, o estudo dos autores De Almeida e Nunes (2018), relatam a importância da atuação interdisciplinar no âmbito no NASF, onde as atividades de grupo realizadas pelo educador físico envolviam sobretudo o incentivo à prática de exercício físico, a partir disso, o mesmo, em conjunto com a equipe desenvolveram atividades lúdicas com abordagem de temas específicos, para serem realizadas no Dia da Mulher e no Dia das Mães. A atividade em comemoração ao Dia da Mulher teve como tema: "Estratégias de alimentação para combater os sintomas da menopausa", onde foi explanado por meio de esclarecimento oral com exposição de cartazes, panfletos informativos com os alimentos que se deve preferir e os que se deve evitar para minimizar os sintomas da menopausa e como atividade em comemoração ao Dia das Mães, foi exposto de uma pirâmide alimentar e realizado uma roda de conversas sobre a importância da alimentação saudável, os alimentos que devem ser mais e menos consumidos e os que precisam ser evitados, sendo possível observar uma participação efetiva no grupo de atividade física, bem como, mudança de hábitos.

Em seguida, o estudo de Novaes e colaboradores (2020), desenvolvido no município de Arapiraca, com os grupos de Práticas Corporais/Atividade Física (PCAF), posteriormente renomeado: "Agite em Casa" foi utilizado como estratégia de reativação dos grupos de atividade física das Academias da Saúde e dos NASF-AB, neste programa foi desenvolvido atividades oferecidas pelos profissionais de educação física, em formato de vídeos curtos que demonstrassem e descrevessem exercícios físicos para membros superiores e membros inferiores, alongamentos dinâmicos, aquecimento, exercícios aeróbicos, exercícios voltados para a musculatura do tronco e imagens explicativas de alongamentos estáticos.

Mediante a pandemia pelo COVID-19, medidas precisaram ser tomadas para se evitar a contaminação em massa, dentre elas, reorganização do processo de trabalho do NASF, corroborando com o trabalho de Novaes e colaboradores (2020), o estudo de De Oliveira e colaboradores (2020) relatam que os atendimentos remotos, passaram a fazer parte do escopo de possibilidades de intervenção do profissional NASF, dessa forma, algumas equipes propuseram a criação de grupos no WhatsApp em cinco linhas: a atenção ao ciclo gravídico-puerperal, atenção à saúde mental, atenção à saúde da criança, atenção à saúde do idoso e atenção em reabilitação. Através da entrada nos grupos e da necessidade de cada usuário serão realizadas estratégias como o monitoramento telefônico e/ou presencial e o repasse de materiais educativos elaborados pela equipe para cada grupo. 
Os resultados de Da Silva e colaboradores (2020), elencaram que os principais grupos realizados em Teresina, Piauí, são os de saúde da mulher, saúde do homem, saúde do idoso, saúde da criança e do adolescente e grupos de gestantes, desses, o de maior adesão são os voltados à saúde da mulher e saúde do idoso, geralmente, durante as atividades de atividades em grupo, são discutidas temáticas que vão variar de acordo com o perfil de cada grupo.

Com relação ao grupo de gestantes, Brito e Gomes, desenvolveram estratégias e atividades que deverão ser realizadas pelos profissionais do NASF do município de Cocal-PI com o objetivo de resolver situações problemas que foram previamente identificadas, tais intervenções baseiam-se em palestras educativas sobre a importância das consultas pré-natais, disseminar informações sobre a importância da vacinação, da reidratação oral e do aleitamento materno, assistência pré natal adequada, simulação de parto humanizado, Rodas de conversa, com trocas de experiências e vivências, suporte psicológico, oficinas de aleitamento materno, palestras sobre alimentação saudável, bem como, na prevenção dos distúrbios nutricionais e das doenças associadas à alimentação e por fim, oficinas e palestras sobre alimentação saudável.

Por fim, o estudo de Bim e colaboradores (2021) retrata que os grupos mais frequentes realizados pela equipe do NASF de Londrina no Paraná, são os grupos de dor e de alongamento, entretanto, há também os grupos de tabagismo, obesidade, ombro, coluna, gestantes, fortalecimento, isostretching, dança terapia e auriculoterapia, além disso, os profissionais relatam que é nos grupos onde há maior "espaço" para a realização de ações de promoção da saúde e de educação em saúde, em razão do vínculo construído nos grupos entre terapeuta e paciente.

No que se refere a auriculoterapia, Saporito, e de Andrade (2017), observaram a necessidade de um curso de capacitação para esta técnica e após a capacitação dos profissionais da equipe do NASF, foi implantado auriculoterapia no grupo de tabagismo para os usuários que demonstrassem interesse, posteriormente, a prática expandiu para outros grupos da unidade de saúde e sendo incorporada em seguida aos grupos de reabilitação, na academia carioca e ao grupo de alimentação, onde obtiveram resultados significativos.

\section{Conclusão}

Observou-se, diante dos resultados obtidos através do presente estudo, que as práticas de grupo realizadas pelas equipes do Núcleo Ampliado de Saúde da Família podem beneficiar inúmeras pessoas, além disso, há a possibilidade de desenvolver grupos nas mais diversas áreas e temas de acordo com as necessidades específicas de cada grupo e consequentemente traçar inúmeras atividades que podem ser propostas para alcançar determinado objetivo. Entretanto, houve certa dificuldade na busca dos artigos, pelo fato de que inúmeros autores apenas citaram o desenvolvimento dos grupos, sem detalhar sua finalidade e quais atividades eram realizadas em cada grupo, deixando uma lacuna que dificulta e compromete o entendimento acerca do processo de trabalho do NASF no que se refere aos grupos terapêuticos/educativos.

Dessa forma, sugere-se que sejam realizados novos estudos com maior riqueza de detalhes acerca das atividades educativas ou terapêuticas realizadas nos mais diversos grupos criados pelas equipes do NASF-AB, favorecendo a compreensão dos próprios profissionais de saúde e da comunidade científica acerca da temática.

\section{Referências}

Aciole, G. G., \& Oliveira, D. K. S. (2017). Percepções de usuários e profissionais da saúde da família sobre o Núcleo de Apoio à Saúde da Família. Saúde em Debate, 41, 1090-1101.

Alves, R. B., Bruning, N. D. O., \& Kohler, K. C. (2019). “O Equilibrista”: Atuação do Psicólogo no NASF no Vale do Itajaí. Psicologia: Ciência e Profissão, 39

Bezerra, A. S. (2021) Análise da autopercepção dos idosos em relação às atividades físicas realizadas no nasf de Jardim do Seridó-RN. Congresso Internacional de envelhecimento humano. 
da Silva, A. D., Nogueira, L. T., Silva, H. G. N., \& Frota, S. C. M. (2020). Atuação do fisioterapeuta nos núcleos de apoio à saúde da família em Teresina, Piauí. Revista Pesquisa em Fisioterapia, 10(4), 648-657.

da Silva, M. I. A., de Alencar Nascimento, J., Garcez, J. C. D., de Castro, N. J. C., \& de Miranda, S. A. (2019). Educação Permanente em Saúde na Estratégia de Saúde da Família: Relato da Elaboração do Plano de Intervenção. Revista Eletrônica Acervo Saúde, 11(16), e1527-e1527.

da Silva, M. E. R., Beirão, A. M., \& Dias, M. S. E. (2018, January). Práticas Integrativas e Complementares da Saúde na promoção de saúde mental: uma parceira entre NASF, ESF e comunidade. In $13^{\circ}$ Congresso Internacional Rede Unida.

DIÁRIO OFICIAL DA UNIÃO. PORTARIA No - 1.742, DE 12 DE JULHO DE 2017. https://pesquisa.in.gov.br/imprensa/jsp/visualiza/index.js p?jornal $=1 \&$ data $=13 / 07 / 2017 \&$ pagina $=124$.

de Almeida, E. A., Silva, A. S. S., \& Nunes, B. V. (2018). Atuação interdisciplinar dos profissionais de nutrição e educação física no âmbito do nasf.

de Moura Santos, D., de Oliveira Monteiro, C. M., \& Damascena, C. G. (2020). A caracterização de grupos de saúde implementados pelas equipes de saúde da família e Nasf-AB em uma regional do Município de Jaboatão dos Guararapes. Research, Society and Development, 9(12), e28891211160-e28891211160.

do Nascimento, D. D. G., \& de Campos Oliveira, M. A. (2010). Reflexões sobre as competências profissionais para o processo de trabalho nos Núcleos de Apoio à Saúde da Família. O mundo da Saúde, 34(1), 92-96.

De Oliveira, Maria Angélica Bezerra et al. A prática do núcleo de apoio à saúde da família do Recife no enfrentamento à pandemia COVID-19. APS em Revista, v. 2, n. 2, p. 142-150, 2020.

de Sousa, L. M. M., Marques-Vieira, C. M. A., Severino, S. S. P., \& Antunes, A. V. (2017). A metodologia de revisão integrativa da literatura em enfermagem. $N^{o} 21$ Série 2-Novembro 2017, 17.

Falci, D. M., \& Belisário, S. A. (2013). A inserção do profissional de educação física na atenção primária à saúde e os desafios em sua formação. InterfaceComunicação, Saúde, Educação, 17, 885-899.

Fernandes, E. T. P., Souza, M. N. D. L., \& Rodrigues, S. M. (2019). Práticas de grupo do Núcleo de Apoio à Saúde da Família: perspectiva do usuário. Physis: Revista de Saúde Coletiva, 29.

Freire, L. P. V., Sales, W. B., de Souza Barbosa, D., \& de Morais, J. D. (2020). As atribuições do fisioterapeuta do Núcleo Ampliado a Saúde da Família e Atenção Básica no município de Lucena-PB. ARCHIVES OF HEALTH INVESTIGATION, 9(1).

Hori, A. A., \& Nascimento, A. D. F. (2014). O Projeto Terapêutico Singular e as práticas de saúde mental nos Núcleos de Apoio à Saúde da Família (NASF) em Guarulhos (SP), Brasil. Ciência \& Saúde Coletiva, 19, 3561-3571.

Leandro-França, C., \& Giardini Murta, S. (2014). Prevenção e promoção da saúde mental no envelhecimento: conceitos e intervenções. Psicologia: Ciência e profissão, 34, 318-329.

MINISTÉRIO DA SAÚDE. DECRETO 7508, DE 28 DE JUNHO DE 2011. Regulamenta a Lei no 8.080, de 19 de setembro de 1990, para dispor sobre a organização do Sistema Único de Saúde - SUS, o planejamento da saúde, a assistência à saúde e a articulação interfederativa, e dá outras providências.: http://www.planalto.gov.br/ccivil_03/_ato2011-2014/2011/decreto/D7508.htm.

MINISTÉRIO DA SAÚDE. Gabinete do Ministro. PORTARIA No 154, DE 24 DE JANEIRO DE 2008. Cria os Núcleos de Apoio à Saúde da Família NASF. Diário Oficial da União. https://bvsms.saude.gov.br/bvs/saudelegis/gm/2008/prt0154_24_01_2008.html.

MINISTÉRIO DA SAÚDE. PORTARIA No 2.436, DE 21 DE SETEMBRO DE 2017. https://bvsms.saude.gov.br/bvs/saudelegis/gm/2017 /prt2436_22_09_2017.html.

Minozzo, F., Kammzetser, C. S., Debastiani, C., Fait, C. S., \& Paulon, S. M. (2012). Grupos de saúde mental na atenção primária à saúde. Fractal: Revista de Psicologia, 24, 323-340.

Moreira, D. C., Soares, D. A., Castro, C. P. D., \& Bispo Júnior, J. P. (2019). Atuação do Núcleo de Apoio à Saúde da Família no fortalecimento da atenção primária: experiências dos agentes comunitários. Physis: Revista de Saúde Coletiva, 29, e290304

Nogueira, I. S., Acioli, S., Carreira, L., \& Baldissera, V. D. A. (2019). Atenção ao idoso: práticas de educação permanente do Núcleo de Apoio à Saúde da Família. Revista da Escola de Enfermagem da USP, 53.

Nogueira, I. S., \& Baldissera, V. D. A. (2018). Educação Permanente em Saúde na atenção ao idoso: dificuldades e facilidades do Núcleo de Apoio à Saúde da Família. Escola Anna Nery, 22.

Quirino, T. R. L., Jucá, A. L., da Rocha, L. P., Cruz, M. S. S., \& Vieira, S. G. (2020). A visita domiciliar como estratégia de cuidado em saúde: reflexões a partir dos Núcleos Ampliados de Saúde da Família e Atenção Básica. Revista sustinere, 8(1), 253-273.

SalesW. B., Cordeiro de OliveiraA. S., PereiraL. E. A., FrançaJ. G. M. de, MarcelinoM. C., GerônimoC. A. da S., ConstatinoA. E. A., SilvaR. B. T. B. da, SilvaR. L. M. da, \& FrançaD. C. M. de. (2020). A importância da equipe NASF/AB - enfretamentos e multidisciplinariedade: uma revisão narrativa/crítica. Revista Eletrônica Acervo Saúde, (48), e3256. https://doi.org/10.25248/reas.e3256.2020

Saporito, B. E., \& de Andrade, G. C. L. (2017) auriculoterapia em atividades coletivas na atenção básica: a experiência de um nasf para ampliação do cuidado em saúde. anais congrepics. campina grande: realize editora

Silva, I. C. B. D., Silva, L. A. B. D., Valença, A. M. G., \& Sampaio, J. (2019). O processo de trabalho do núcleo ampliado de saúde da família e atenção básica. Trabalho, Educação e Saúde, 17.

Soares, P. H. S., \& de Jesus Santos, L. O NASF-AB como articulador de grupos educativos na unidade básica de saúde: relato de experiência do estágio curriculaR 
Research, Society and Development, v. 10, n. 17, e83101724497, 2021

(CC BY 4.0) | ISSN 2525-3409 | DOI: http://dx.doi.org/10.33448/rsd-v10i17.24497

Souza, F. L. D., Chacur, E. P., Martins, R. C. C., Silva, L. A. M., Góes, R. W. L., \& Vilela, W. V. (2013). Percepções sobre qualidade de vida das usuárias de um núcleo de apoio à saúde da família. Investigação, 13(3), 34-38.

Souza, T. S., \& Medina, M. G. (2018). Nasf: fragmentação ou integração do trabalho em saúde na APS? Saúde em Debate, 42, 145-158.

Totoro, R., Sousa, M., Martins, R., Chacur, E., \& Souza, F. (2017). Avaliação da qualidade de vida dos usuários de um núcleo de apoio à saúde da famíliaNASF. Psicologia e Saúde em debate, 3(1), 50-67.

Vendruscolo, C., Trindade, L. D. L., Metelski, F. K., Vandresen, L., Pires, D. E. P. D., Tesser, C. D., \& Martins, M. M. F. P. D. S. (2020). Contribuições da educação permanente aos núcleos ampliados de saúde da família. Escola Anna Nery, 24. 